\title{
cis- and trans-Acting Elements Involved in Amino Acid Regulation of Asparagine Synthetase Gene Expression
}

\author{
LUISA GUERRINI, $\uparrow$ SHIH S. GONG, KAREN MANGASARIAN, AND CLAUDIO BASILICO* \\ Department of Microbiology and Kaplan Cancer Center, New York University School of Medicine, \\ New York, New York 10016
}

Received 26 October 1992/Returned for modification 6 January 1993/Accepted 6 March 1993

\begin{abstract}
We have previously shown that asparagine synthetase (AS) mRNA expression can be dramatically upregulated by asparagine deprivation in ts11 cells, mutants of BHK hamster cells which encode a temperaturesensitive AS. The expression of AS mRNA was also induced upon starvation for one of several essential amino acids in HeLa cells. We also showed that regulation of AS mRNA expression by amino acid concentration has both transcriptional and posttranscriptional components. Here we report the analysis of the elements in the human AS promoter region important for its basal activity and activation by amino acid starvation. Our results indicate that a DNA fragment spanning from nucleotides -164 to +44 of the AS promoter is sufficient for uninduced and induced gene expression. Mutations in a region located 15 to 30 bp downstream from the major transcription start site that shows good homology to a sequence in the first exon of c-fos implicated as a negative regulatory element resulted in a significant increase in basal gene expression but did not affect regulation. Interestingly, this region binds single-stranded-DNA-binding proteins that are specific for the AS coding strand. Mutations in either one of two putative binding sites for transcription factor Sp1, in a region of approximately 60 bp where many minor RNA start sites are located, or at the major transcription start site decreased promoter activity, but significant induction by amino acid starvation was still observed. Strikingly, mutations centered around nucleotide -68 not only decreased the basal promoter activity but also abolished amino acid regulation. This DNA region contains the sequence $5^{\prime}$-CATGATG-3', which we call the amino acid response element (AARE), that can bind a factor(s) present in HeLa cells nuclear extracts that is not capable of binding to an AS promoter with mutations or deletions of the AARE. This finding is in line with the hypothesis that transcriptional activation of AS gene expression is mediated through the binding of a positive regulatory element. We did not detect changes in the level of binding of this factor to the AARE by using nuclear extracts from HeLa cells grown under starved conditions, suggesting that activation of this factor(s) results from posttranslational modification or complexing with other proteins that do not affect its DNAbinding properties.
\end{abstract}

The signals regulating gene expression in prokaryotes and lower eukaryotes in response to nutrient variation are well known and characterized; it is well established that these organisms are quickly able to adjust to variations in nutrient supply and to intracellular amino acid levels by altering their patterns of gene expression. Much less is known about the response of higher eukaryotes to nutrient deprivation (9).

We have previously shown $(13,15)$ that the expression of the human and hamster asparagine synthetase (AS) gene is regulated by the intracellular concentration of Asn in ts11 cells, temperature-sensitive growth mutants of the BHK hamster cell line that owe their phenotype to the production of a nonfunctional AS at the nonpermissive temperature. Exposure of ts 11 cells to $39.5^{\circ} \mathrm{C}$ leads to a dramatic increase of AS mRNA expression, and this induction can be prevented by addition of exogenous Asn to the culture media, indicating that AS gene expression is regulated by the intracellular levels of Asn. AS mRNA expression can also be induced by starvation for several essential amino acids in wild-type BHK and HeLa cells (13). The finding that starvation for a variety of amino acids has the same effects as Asn deprivation on AS mRNA expression is reminiscent of the Saccharomyces cerevisiae yeast system, in which sev-

\footnotetext{
* Corresponding author.

$\dagger$ Present address: Department of Genetics and Biology of Microorganisms, University of Milan, Milan, Italy.
}

eral biosynthetic pathways are induced by starvation for different amino acids through the activation of the transcription factor GCN4 $(17,18)$.

The RNA levels of transcription factors such as c-fos, c-jun, and c-myc have been reported to be induced transcriptionally or posttranscriptionally by amino acid deprivation, but the changes observed were rather slight (30). Since no general accumulation of mRNAs in amino acid-starved cells has been observed $(13,30)$, animal cells must have a specific mechanism that enables them to alter their pattern of gene expression in response to amino acid deprivation.

Regulation of AS expression by amino acid starvation has transcriptional and posttranscriptional components (13); in ts 11 cells, under conditions of Asn starvation, the AS mRNA half-life is very long (more than $12 \mathrm{~h}$ ), but the addition of Asn to the culture media shortens the AS mRNA half-life to less than $2 \mathrm{~h}$. This effect is blocked by inhibitors of transcription and translation, indicating that restoration of normal amino acid levels induces the expression of a nuclease involved in the down regulation of AS mRNA levels. We also showed that the levels of AS mRNAs with a shortened open reading frame were not regulated by amino acid starvation, suggesting that translation is required for the regulation of AS mRNA stability (13). On the other hand, when the same AS mRNAs were placed under the control of a 3.4-kb DNA fragment containing the human AS promoter region, a significant degree of regulation was observed, indicating that AS mRNA induction by amino acid starvation is also under 
transcriptional control. Accordingly, chloramphenicol acetyltransferase (CAT) gene expression was regulated by amino acid concentration when placed under the control of the AS gene $5^{\prime}$ region (13).

We report here the characterization of the cis-acting elements involved in the constitutive activity and in transcriptional regulation of the AS promoter. By deletion analysis of the AS 3.4-kb 5' region, we were able to localize these elements to a DNA fragment spanning from nucleotides -164 to +44 . Within this region are sequences which are important for constitutive expression as well as a binding site (amino acid-response element [AARE]) for a protein(s) that appears to be the mediator of the transcriptional activation of AS gene expression in response to amino acid starvation. Deletions or mutations in this region resulted in low and uninducible levels of gene expression, indicating that transcriptional activation of AS gene expression in response to amino acid starvation is mediated through the binding of a transcriptional activator to the AARE.

\section{MATERIALS AND METHODS}

Cell culture. Cells were cultured in Dulbecco's modified Eagle's medium (DMEM) containing $10 \%$ calf serum. When indicated, DMEM was supplemented with asparagine (50 $\mu \mathrm{g} / \mathrm{ml})$. When indicated, DMEM lacking leucine or glutamine was used. The concentrations of those amino acid in control medium were $104.8 \mu \mathrm{g} / \mathrm{ml}$ (leucine) and $60 \mu \mathrm{g} / \mathrm{ml}$ (glutamine). ts 11 cells were maintained at $33^{\circ} \mathrm{C}$, and $\mathrm{HeLa}$ cells were maintained at $37^{\circ} \mathrm{C}$. In all experiments involving amino acid starvation, $10 \%$ dialyzed calf serum was used.

DNA transfection and CAT assay. HeLa cells $\left(5 \times 10^{5}\right)$ and ts 11 cells $\left(10^{6}\right)$ were plated in 100 -mm-diameter dishes. The next day, the cells were refed with fresh medium $4 \mathrm{~h}$ before the transfection by the calcium phosphate coprecipitation method as previously described $(13,15)$. Ten micrograms of CAT plasmid was transfected into the cells along with $2 \mu \mathrm{g}$ of pCH110 (16), a plasmid containing the bacterial $\beta$-galactosidase gene fused to the simian virus 40 promoter, as an internal control. The cells were exposed to the precipitate for $5 \mathrm{~h}$ in the presence of $10 \mu \mathrm{M}$ chloroquine and then shocked with $15 \%$ glycerol in HEPES-buffered saline (HBS) for $3 \mathrm{~min}$ at room temperature. At $24 \mathrm{~h}$ after the glycerol shock, cells were amino acid starved for $14 \mathrm{~h}$ and then collected for CAT assay (14). $\beta$-Galactosidase activity was measured (16), and in most cases, cell lysates containing 0.1 $\mathrm{U}$ of $\beta$-galactosidase were analyzed for CAT activity.

RNA preparation and analysis. For RNase protection assay, a 250-bp EcoRI-HindIII fragment of the CAT gene from plasmid pSV2CAT (14) was subcloned in Bluescript at the SmaI site. Sense and antisense RNA probes were generated by first linearizing the plasmids with BamHI or EcoRI and then transcribing them with T3 or T7 RNA polymerase, respectively, in the presence of $\left[\alpha-{ }^{32} \mathrm{P}\right] \mathrm{CTP}$. Total RNA was extracted from transiently transfected ts 11 cells by the guanidine-isothiocyanate method (33), and $10 \mu \mathrm{g}$ was analyzed by the RNase protection assay according to Melton et al. (24). Primer extension analysis of RNA was performed as described previously (15).

Plasmid and mutant construction. Plasmid p3.4CAT, containing the CAT gene under the control of a 3.4-kb fragment of the human AS 5 ' region, was as described previously (15). Plasmids p1.8CAT, p1.1CAT, p0.4CAT, p0.97CAT, p0.85CAT, and p0.83CAT, which contain various deletions within the 3.4-kb AS DNA fragment, were constructed according to standard recombinant DNA techniques (33). A 600-bp BstEII-HindIII fragment of the human AS gene, containing part of the second intron and the first $12 \mathrm{bp}$ of the third exon, was cloned into the HindIII site of the pSV0CAT vector (14). The unique KpnI site contained in this AS fragment was used for cloning all deletion mutants of the 3.4-kb AS fragment obtained by restriction enzyme digestion.

To construct plasmids p0.3CAT, p0.3CAT with a 16-bp internal deletion, and mutants in the AS first-exon region that shares homology with the fos-inducible response element (FIRE) sequence, DNA fragments containing the desired mutations were generated by the polymerase chain reaction (PCR) technique. Briefly, the 1.1-kb HindIII-SphI fragment of the AS promoter region was subcloned in the pGEM3 vector as the template for the PCRs. To generate the mutants, an oligonucleotide contained in the $\mathrm{PGEM}$ vector $\mathrm{T} 7$ promoter region was used as the $5^{\prime}$ primer in all cases along with an oligonucleotide of the AS region containing the desired mutation as the $3^{\prime}$ primer. The sequences of the mutagenic $3^{\prime}$ primer are as follows (a KpnI site is underlined): TATGGTACCTCCTAAGCAGGTCAGGGTGATGT GGCGGGCTGtcGCCAGGGAT, 2-bp substitution in the AS first exon; TATGGTACCTCCTAAGCAGGTCAGGGTGA TGTGGCGGGCTGAGGTGTGGACAGC, 10-bp deletion in the AS first exon; TATGGTACCTCCTGTAACGCGTGC GGG, p0.3CAT; and TATGGTACCCCTGGCTCCTGTAA CGGCGTGCGGCGCGAAGTTGACCAACCAGCGCGCA, a 16-bp internal deletion in p0.3CAT. Each oligonucleotide also contains a KpnI site at the $5^{\prime}$ end, and the fragments produced by PCR were digested with $K p n I$ and purified. The mutated DNA fragments were cloned into the unique KpnI site of the vector described above. The orientation of the inserts was determined by restriction enzyme digestions, and the presence of the desired mutations was determined by sequence analysis (34).

Plasmids MS1 to MS14, $\Delta 8$, and $\Delta 16$ were constructed by site-directed mutagenesis by the method of Kunkel (21). The sequences of the oligonucleotides were as follows (lowercase letters denote mutant substitutions): MS1, GGACTA AGTaaaattttGGAAGGGCGC; MS2, GGGCGGAGCcttcagc cGTGGGGCGGCG; MS3, CGGGGTGTCaaaaataaAGCAG GACTA; MS4, GGGCGGGGCCtattttgtTCCGGGGCGGA; MS5, AGCGCGCACAtttattttCCGCGGGGTG; MS6, GAG GACCAACactatataACAGGGCGGG; MS7, TCATGCCTG CtcttcaacACCAGCGCG; MS8, GGAAGTTTCAgacgtaagG CGAGGACC; MS9, ACGCGTGCGttcetgggCATCATGCCT; MS10, GGCTCCTGTccatatgtCGGGAAGTTT; MS11, ATA CCGACCgttagaagGTAACGCGT; MS12, CGTGGCGCTgcg caatcCCTGGCTCCT; MS13, GCGGCGAGGaatcttatCTTA TACCGA; MS14, GGATGTGGAactaggtcCGGGCGGCGA; $\Delta 8$, AACGCGTGCGGGAAGTTCCTGCGAGGACCAAC CA; and $\triangle 16$, AACGCGTGCGGGAAGTTGACCAACCAG CGCGC. The fragments containing the desired mutations generated by this method were used to replace the corresponding wild-type sequences in plasmid p3.4CAT.

Electrophoretic mobility shift assay. Total nuclear extracts were prepared from HeLa cells grown in suspension in DMEM or DMEM lacking leucine by the method of Dignam et al. (10). Single-stranded oligonucleotide probes were labeled with ${ }^{32} \mathrm{P}$ by $\mathrm{T} 4$ polynucleotide kinase, and doublestranded probes were labeled by filling in with Klenow enzyme. Binding reactions were carried out in $15 \mu \mathrm{l}$ of 10 $\mathrm{mM} N$-2-hydroxyethylpiperazine- $N^{\prime}$-2-ethanesulfonic acid (HEPES; pH 7.9)-10\% (vol/vol) glycerol-50 mM KCl-0.1 mM EDTA- $-0.5 \mathrm{mM}$ phenylmethylsulfonyl fluoride- 0.5 or $2.5 \mathrm{mM}$ dithiothreitol. After incubation of $10 \mu \mathrm{g}$ of nuclear 
TABLE 1. Regulation of CAT activity under the control of the AS $5^{\prime}$ region in amino acid-starved cells ${ }^{a}$

\begin{tabular}{|c|c|c|c|c|c|c|}
\hline \multirow{3}{*}{ Plasmid } & \multicolumn{6}{|c|}{ Avg $\%$ of acetylated $\left[{ }^{14} \mathrm{C}\right]$ chloramphenicol $\pm \mathrm{SE}^{b}$} \\
\hline & \multicolumn{3}{|c|}{ HeLa cells } & \multicolumn{3}{|c|}{ ts11 cells } \\
\hline & +Leu & -Leu & -Leu/+Leu & + Asn & - Asn & $-A s n /+$ Asn \\
\hline $\begin{array}{l}\text { p3.4CAT (AS) } \\
\text { pSV2CAT }\end{array}$ & $\begin{array}{r}5 \pm 0.7 \\
20.4 \pm 2.6\end{array}$ & $\begin{array}{l}52.8 \pm 5.9 \\
13.2 \pm 2.1\end{array}$ & $\begin{array}{c}10.6(16.2) \\
0.65(1)\end{array}$ & $\begin{aligned} 7.4 & \pm 1.9 \\
19.4 & \pm 7.1\end{aligned}$ & $\begin{aligned} 43 & \pm 10.3 \\
11.8 & \pm 4.9\end{aligned}$ & $\begin{array}{l}5.8(9.6) \\
0.6(1)\end{array}$ \\
\hline
\end{tabular}

${ }^{a}$ ts 11 and HeLa cells were transiently transfected with plasmid p3.4CAT containing the AS 5 ' region or with plasmid pSV2CAT along with plasmid pCH110 carrying the $\beta$-galactosidase gene. HeLa cells were maintained at $37^{\circ} \mathrm{C}$ throughout the experiments, while ts 11 cells were transfected at $33^{\circ} \mathrm{C}$ and shifted to $39.5^{\circ}$ $24 \mathrm{~h}$ later. Transfection and CAT assays were performed as described in Materials and Methods. In all cases, an amount of cells extract equivalent to 0.1 Unit of $\beta$-galactosidase was used.

${ }^{b}$ The data represent averages of five independent experiments. In parentheses is the ratio of CAT activity under starved versus nonstarved conditions normalized for the values obtained for pSV2CAT.

extracts for $5 \mathrm{~min}$ on ice with $2 \mu \mathrm{g}$ of poly $(\mathrm{dI}-\mathrm{dC})$ in sample buffer, $0.5 \mathrm{ng}$ of probe and cold competitor, as indicated, was added, and incubations were continued for $20 \mathrm{~min}$ at room temperature. Samples were then run on a $5 \%$ native polyacrylamide gel in $0.2 \times$ Tris-borate-EDTA.

\section{RESULTS}

Expression from the AS $5^{\prime}$ region is regulated by amino acid levels in ts11 and HeLa cells. We previously showed (13) that elements contained in the $5^{\prime}$ region of the AS gene are involved in the induction of AS mRNA expression by amino acid starvation. A 3.4-kb fragment of the human AS 5' region, containing the AS promoter, the first two untranslated exons and introns, and $12 \mathrm{bp}$ of the third exon (15), was fused to the CAT gene to generate plasmid p3.4CAT and stably transfected into ts 11 cells. Exposure of the stable ts 11 transformants to the nonpermissive temperature of $39.5^{\circ} \mathrm{C}$, at which their AS is nonfunctional, resulted in increased levels of CAT mRNA, and this increase was prevented by the addition of exogenous Asn. To identify the cis-acting elements involved in basal activity and in the induction of AS gene expression by amino acid starvation, we first transiently transfected plasmid p3.4CAT into ts11 cells and HeLa cells, which were then exposed to conditions of Asn and Leu starvation, respectively. In all experiments, $10 \mu \mathrm{g}$ of CAT plasmid was cotransfected with $2 \mu \mathrm{g}$ of pCH110, which contains the $\beta$-galactosidase gene fused to the simian virus 40 promoter (16). The levels of $\beta$-galactosidase were measured, and $0.1 \mathrm{U}$ of $\beta$-galactosidase was used for each CAT reaction to normalize for transfection efficiency and for the possible differential translation of the CAT protein in the starved versus nonstarved conditions. As shown in Table 1, the levels of CAT protein expressed under the control of the AS 5 ' region are induced severalfold by amino acid starvation in a transient assay both in ts11 (-Asn) and HeLa (-Leu) cells compared with nonstarved conditions, whereas the CAT protein levels expressed from the pSV2CAT construct used as a control were not induced but were actually slightly lowered by amino acid starvation. We observed a 10to 16-fold induction for the AS-controlled CAT activity, after normalization for the differences seen with the pSV2CAT construct, in the different conditions, despite the fact that the same amounts of $\beta$-galactosidase units were used in both reactions. The results obtained from transient transfection of p3.4CAT into ts 11 and HeLa cells were in agreement with those obtained from the stable ts11 transformants (13) and also showed that in both cell lines, regulation by amino acid starvation is mediated through the same $3.4-\mathrm{kb}$ fragment.

We wanted to determine whether under our experimental conditions amino acid starvation affected translation of the CAT mRNA, and we also wanted to directly measure the amounts of CAT RNAs transcribed under starved and nonstarved conditions. We therefore transiently transfected plasmids p3.4CAT and pSV2CAT into ts 11 cells at $33^{\circ} \mathrm{C}$ and $24 \mathrm{~h}$ later shifted the cells to $39.5^{\circ} \mathrm{C}$ for an additional $24 \mathrm{~h}$ with or without exogenous Asn $(50 \mu \mathrm{g} / \mathrm{ml})$. Total RNA was extracted, and $10 \mu \mathrm{g}$ was analyzed by the RNase protection method using an antisense CAT probe. As shown in Fig. 1A, under starvation conditions, the levels of CAT mRNA protected by the antisense probe are much higher (lane 3 ) than those protected in normal conditions (lane 1) for

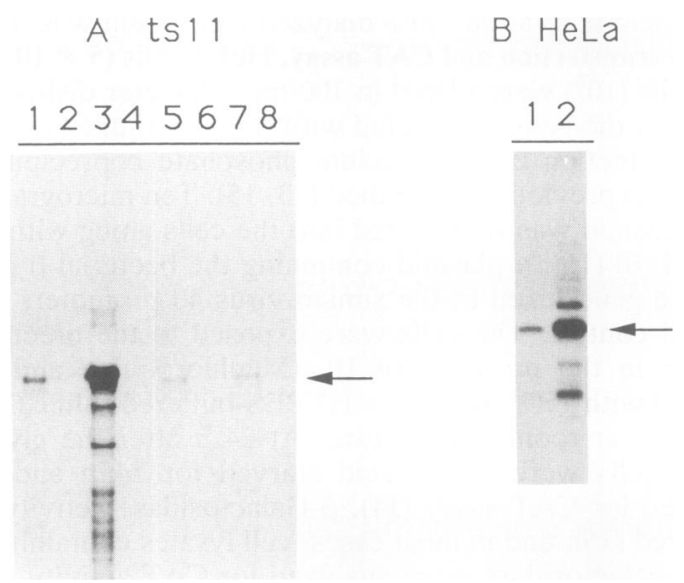

FIG. 1. Expression of CAT mRNA under the control of the AS 5 region. (A) ts 11 cells were transiently transfected with plasmid p3.4CAT (lanes 1 to 4 ) or pSV2CAT (lanes 5 to 8 ). At $24 \mathrm{~h}$ after transfection, cells were exposed for $14 \mathrm{~h}$ to the nonpermissive temperature $\left(39.5^{\circ} \mathrm{C}\right)$ with (lanes $1,2,5$, and 6) or without (lanes 3 , 4,7 , and 8) exogenous Asn added to the media $(50 \mu \mathrm{g} / \mathrm{ml})$. Cells were harvested, and total RNA was extracted; $10 \mu \mathrm{g}$ was analyzed for CAT RNA expression by the RNase protection assay as described in Materials and Methods, using an antisense CAT RNA probe (lanes $1,3,5$, and 7) or a sense CAT RNA probe (lane 2, 4, 6, and 8 ) as a control. The arrow indicates the protected probe. (B) $\mathrm{HeLa}$ cells were transfected with $10 \mu \mathrm{g}$ of plasmid p3.4CAT. At 24 $\mathrm{h}$ after transfection, cells were incubated in DMEM containing $10 \%$ dialyzed calf serum (lane 1) or DMEM lacking leucine and containing $10 \%$ dialyzed calf serum (lane 2 ) for $14 \mathrm{~h}$, and total cellular RNA was extracted; $40 \mu \mathrm{g}$ of each sample was analyzed for CAT RNA expression by primer extension assay as described in Materials and Methods, using an oligonucleotide with the sequence $5^{\prime}$-CGTA ATATCCAGCTGAACGGT-3' contained in the CAT coding region. Each arrow indicates the CAT RNA initiated from the AS major start site. 


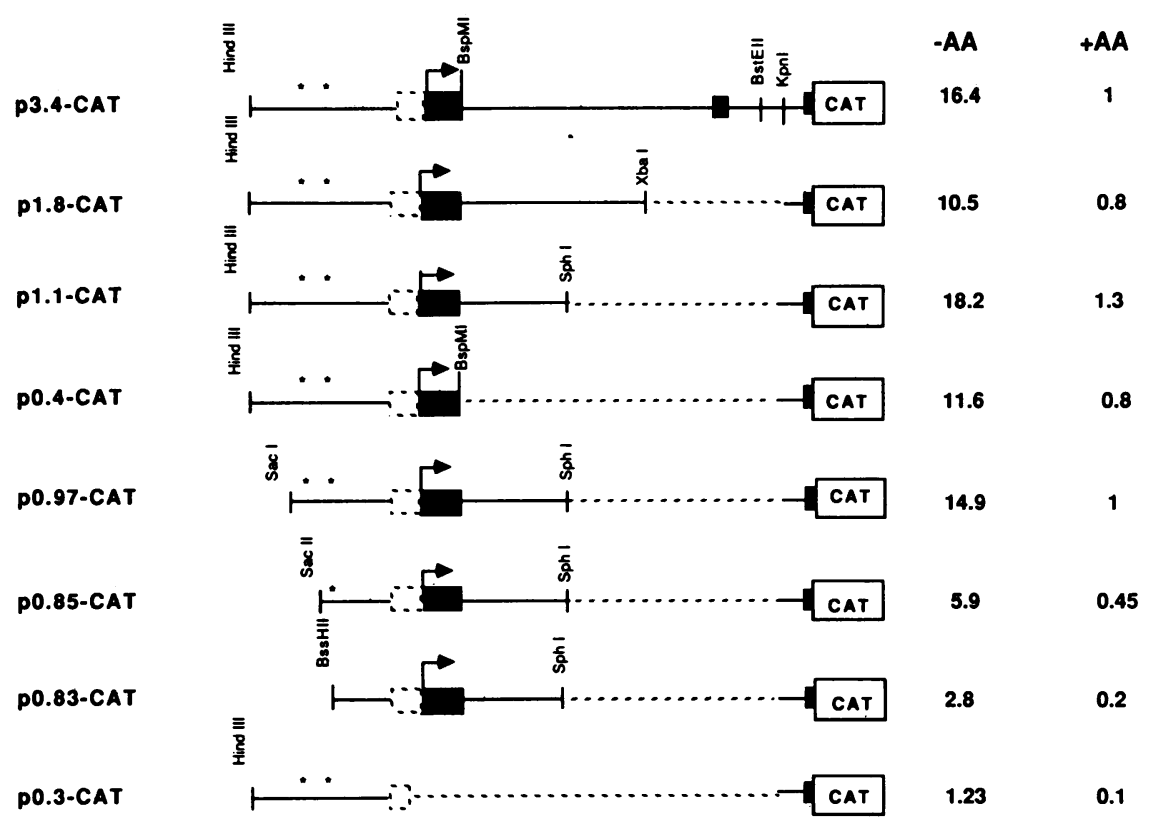

FIG. 2. Identification of the AS DNA region regulated by amino acid starvation. Shown are schematic representations (not to scale) of the plasmid constructs used for transfection. The plasmids were constructed as described in Materials and Methods. Solid box, exon; arrow, major transcription start site; solid line, upstream promoter region or intron; dashed line, deletion; dashed box, minor transcription start site; asterisks, 5'-CCGCCC-3' sequence. Plasmid p0.3CAT contains AS upstream sequences spanning from -340 to -35 , with the nucleotide number of the major transcription start site designated +1 . HeLa cells were transfected with each of the plasmids shown together with a $\beta$-galactosidase gene plasmid, pCH110, as described in Materials and Methods; $24 \mathrm{~h}$ after transfection, cells were incubated in fresh DMEM (+AA) containing $10 \%$ dialyzed serum or DMEM lacking leucine (-AA) and containing $10 \%$ dialyzed serum for $14 \mathrm{~h}$. $\beta$-Galactosidase and CAT activities were determined as described in Materials and Methods. The numbers represent CAT activities of the plasmids in nonstarved $(+\mathrm{AA})$ or starved $(-\mathrm{AA})$ cells relative to that of plasmid p3.4CAT in nonstarved conditions and are the averages of several independent experiments.

p3.4CAT, while the levels of CAT mRNA derived from pSV2CAT are unchanged (lanes 5 and 7). The sense probe did not protect any RNA species (lane $2,4,6$, and 8 ). Transfection of the same plasmids into $\mathrm{HeLa}$ cells and measurement of CAT RNA by primer extension following leucine starvation gave essentially the same results (Fig. 1B). These data showed that the degree of regulation seen on expression of the p3.4CAT mRNAs, which we estimate to be about 12-fold, is in agreement with the degree of regulation determined in the CAT assays and confirmed the results previously obtained (13) for ts 11 cells that were stably transfected with plasmid p3.4CAT.

Identification of the minimum AS DNA region regulated by amino acid starvation. The 3.4-kb fragment of the human AS gene used in the previous experiments included the AS promoter region, the first two untranslated exons and introns, and $12 \mathrm{bp}$ of the third exon. To determine whether transcriptional or posttranscriptional events were responsible for the induction seen with the 3.4-kb AS fragment, we introduced progressive deletions from the $5^{\prime}$ end and from the $3^{\prime}$ end, using restriction enzyme sites. The basal and inducible activities of the CAT deletion mutants were tested by transient transfection into HeLa cells. As shown in Fig. 2, deletion of the second untranslated exon and part of the first intron between $X b a \mathrm{I}$ and $K p n I$ sites (p1.8CAT) had no major effect on basal line activity and on the inducibility of the constructs by amino acid starvation. Similar results were obtained by further deletions of the first intron (p1.1CAT and p0.4CAT). Deletions of the first $186 \mathrm{bp}$ from the $5^{\prime}$ end by cleavage with SacI (p0.97CAT) had no effect either on basal activity or on inducibility by amino acid starvation; further deletions eliminating either one or two of the Sp1 binding sites contained in the AS promoter region lowered the baseline activity of these constructs, while they had no effect on the regulation by amino acid levels. Deletion of one Sp1 binding site by cleavage with SacII (p0.85CAT) lowered the CAT activity by about $60 \%$ compared with that of p3.4CAT, and deletion of both $\mathrm{Sp1}$ binding sites by cleavage with BssHII (p0.83CAT) lowered it further. These results are in agreement with the finding of cooperativity between multiple Sp1 binding sites on the activity of certain promoters (3). Even though the basal activities of constructs $\mathrm{p} 0.85$ and p0.83 CAT were lower than that of p3.4 CAT, similar levels of inducibility by amino acid starvation were observed. From these results, we concluded that the minimum AS promoter retaining full activity starts at $-164 \mathrm{bp}$ from the major transcription start site and that the two Sp1 binding sites are required for baseline activity and not for inducibility by amino acid starvation. The minimum AS region retaining full activity and inducibility by amino acid starvation spans from -164 to +44 (between the BssHII and the BspMI sites) of the AS gene. Similar results were obtained with transfection into ts 11 cells (data not shown).

Elements downstream of the major transcription start site. Others genes such as fos, jun, myc, and $O D C$ have been reported to be induced by amino acid starvation (30). Sequence comparisons between these genes and the $5^{\prime}$ portion of the AS gene revealed an interesting similarity; all of them had an untranslated first exon which contained a region that had significant homology with the FIRE sequence (22). The comparison between the AS and fos sequences is shown in Fig. 3A. The FIRE sequence was initially identified as the 


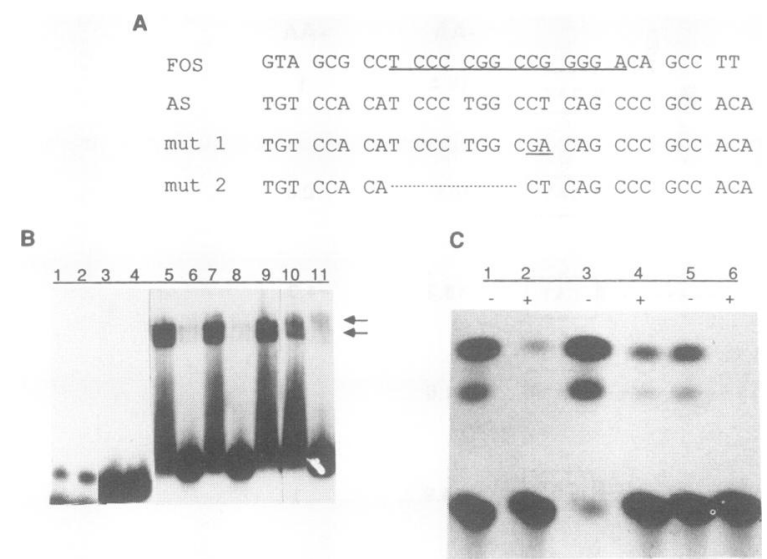

FIG. 3. Elements downstream of the major transcription start site. (A) Sequence comparison of the FIRE (underlined) region with the homologous sequences in the AS +5 to +35 region. mut 1 and mut 2 contain 2- and 8-bp substitutions (underlined), respectively, within the FIRE homology region. All of the sequences shown are for the coding strand (mRNA strand). (B) Electrophoretic mobility shift assays performed with labeled double-stranded AS +5/+35 oligonucleotide probes as shown in panel A (lanes 1 and 2), AS $+5 /+35$ noncoding (lanes 3 and 4 ) or coding (lanes 5 to 9) strand, mut 1 coding strand (lane 10), or mut 2 coding strand (lane 11). Nuclear extracts were prepared from $\mathrm{HeLa}$ cells grown in nonstarved conditions (lanes $1,3,5,6,10$, and 11 ) or starved for $5 \mathrm{~h}$ in medium lacking leucine (lanes $2,4,7,8$, and 9). Reactions in lanes 6 and 8 were the same as those in lanes 5 and 7 , respectively, but were competed for with excess unlabeled single-stranded AS +5/ +35 coding strand. The reaction in lane 9 was the same as that in lane 7 but was competed for with excess AS $+5 /+35$ noncoding strand. The arrows indicate specific DNA-protein complexes. (C) mut 1 and mut 2 (see panel A) were introduced in plasmid p0.4CAT as described in Materials and Methods. After transfection in HeLa cells, CAT gene expression was measured in cells grown in starved $(-)$ and nonstarved $(+)$ conditions. The autoradiograph of a representative thin-layer chromatograph of the CAT assays is shown. Lanes: 1 and 2, p0.4CAT mut 1;3 and 4: p0.4CAT mut 2; 5 and 6, p0.4CAT. Quantitation of CAT activity gave the following results (percent acetylated chloramphenicol); lane 1, 34; lane 2, 2.5; lane 3, 90; lane 4, 8; lane 5, 13; and lane 6,1 . In this assay, an amount of extract corresponding to $0.05 \mathrm{U}$ of $\beta$-galactosidase was used.

target site for the binding of a regulatory protein of fos gene expression. We thus attempted to determine whether any protein bound to the AS sequences in the DNA region homologous to the FIRE sequence. Two oligonucleotides covering the region of homology were synthesized, annealed, and used as double-stranded probes in gel mobility shift experiments (Fig. 3B). HeLa nuclear cells extracts from starved and nonstarved celis (lanes 1 and 2) did not give rise to any retarded band. When the same oligonucleotides were used separately as single-stranded DNA probes, the noncoding strand did not give any retarded band (lanes 3 and 4), while the coding strand retarded two bands (lane 5) that were competed for by excess specific oligonucleotides (lane 6) and not by nonspecific oligonucleotides (not shown). The two retarded bands did not change either in abundance or in relative ratio with amino acid starvation (compare lanes 5 and 7). The noncoding probe did not compete for binding (lane 9), and we could not detect any binding to (or competition by) a sense RNA probe spanning the same region (not shown).

To understand the role of these proteins in AS gene expression, we mutated the DNA sequence to abolish bind-

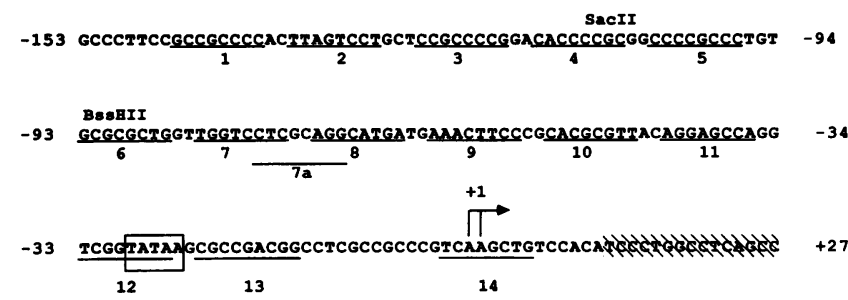

+28 CGCCACATCACCCTGACCTGCTTACGCCCAGgtgagecgggt

FIG. 4. Sequence of the AS promoter and first exon. The arrow indicates the major transcription start site, whose nucleotide number is designated +1 . The lowercase letters indicate intronic sequences. The underlined regions designated by numbers 1 to 14 indicate the regions of the substitution mutations contained in the corresponding MS-CAT plasmids. The mutant sequences are described in Materials and Methods. A TATAA sequence is indicated by a box. The shaded region contains sequences sharing homology with the FIRE sequence (Fig. 3).

ing to these proteins. A two-base substitution considerably diminished the binding affinity, while an internal eight-base deletion abolished it almost completely (Fig. 3B, lanes 10 and 11). We then introduced by PCR these mutations in the p0.4CAT construct, which has the minimum AS promoter and the first exon (Fig. 2). The activities of the constructs are shown in Fig. 3C. Both mutants, particularly the deletion, had basal CAT activity higher than that of the wild type but were still regulated by amino acid deprivation. From these data, we conclude that these single-stranded-DNA-binding proteins play no major role in AS gene regulation by amino acid starvation and that the binding of these proteins to the AS coding strand had a repressing effect on AS gene expression. Similar inhibitory roles for single-strandedDNA-binding proteins on gene expression have been reported for other genes (28).

Elements upstream of the major transcription start site. Since no role in amino acid regulation of the AS promoter was found for the two single-stranded-DNA-binding proteins described above, we investigated the role of the major transcription start site and that of a TATAA sequence, which is present approximately 25 nucleotides upstream of the major transcription initiation site (Fig. 4), in AS gene expression. A TATAA deletion mutant was regulated by amino acid starvation, but it was about $20 \%$ less active than the parental construct, and similar results were obtained with a deletion centered around the major transcription start site (not shown). To confirm that the region encompassing the TATAA sequence and the major transcription start site was not required for the transcriptional regulation of AS gene expression, another mutant was generated by PCR. As shown in Fig. 2, this construct (p0.3CAT) contains sequences spanning from -340 to -35 and lacks both the TATA box and the major transcription start site; the basal level of activity of this construct was very low, but it was detectable with use of five times more $\beta$-galactosidase units than was usually used. This plasmid was regulated as well as p3.4CAT by amino acid starvation, thus narrowing the region required for the amino acid response from -93 (p0.83CAT; Fig. 2) to -35 .

Mutational analysis of the -150 to +1 region of the AS promoter. The results of the experiments described so far had shown that transcriptional regulation of the AS gene was under the control of multiple factors and had tentatively identified the DNA sequences spanning nucleotides -93 to 
TABLE 2. Mutational analysis of the -150 to +1 region of the AS promoter ${ }^{a}$

\begin{tabular}{lclc}
\hline \multirow{2}{*}{ Plasmid } & \multicolumn{2}{c}{ Relative CAT activity } \\
\cline { 2 - 4 } & $-\mathrm{AA}$ & $+\mathrm{AA}$ & $-\mathrm{AA} / \mathrm{AA}$ \\
\hline p3.4-CAT & 17.0 & 1.0 & 17.0 \\
MS1-CAT & 20.0 & 1.66 & 12.0 \\
MS2-CAT & 17.0 & 2.3 & 7.4 \\
MS3-CAT & 6.6 & 0.66 & 10.0 \\
MS4-CAT & 21.6 & 2.0 & 10.8 \\
MS5-CAT & 5.3 & 0.5 & 10.0 \\
MS6-CAT & 17.6 & 1.5 & 11.7 \\
MS7-CAT & 8.3 & 0.66 & 12.6 \\
MS7a-CAT & 10.0 & 1.3 & 7.7 \\
MS8-CAT & 0.4 & 0.66 & 0.6 \\
MS9-CAT & 1.8 & 0.5 & 3.6 \\
MS10-CAT & 3.3 & 0.66 & 5.0 \\
MS11-CAT & 3.6 & 0.66 & 6.0 \\
MS12-CAT & 4.0 & 0.33 & 12.0 \\
MS13-CAT & 5.0 & 0.66 & 8.0 \\
MS14-CAT & 3.0 & 0.33 & 9.0 \\
$\Delta 16-C A T$ & 0.1 & 0.29 & 0.35 \\
$\Delta 8-C A T$ & 0.16 & 0.33 & 0.49 \\
pSV2CAT & 5.3 & 7.3 & 0.7 \\
\hline
\end{tabular}

${ }^{a}$ Plasmid constructs MS1 to MS14 each contained an 8-bp substitution along the -150 to +1 region of the AS promoter (Fig. 4) in p3.4CAT. Plasmids $\Delta 8$ and $\Delta 16$ contained internal deletions spanning from -70 to -63 and -78 to -63 , respectively, of the AS promoter in plasmid p3.4CAT. CAT gene expression of each plasmid was analyzed by transfecting $\mathrm{HeLa}$ cells and determining CAT activities in amino acid-starved (-AA) or nonstarved (+AA) conditions as described in Materials and Methods.

${ }^{b}$ CAT activity of each plasmid relative to that of plasmid p3.4CAT in nonstarved HeLa cells; average of two independent experiments.

-35 as the most important region for regulation by amino acid concentration. To pinpoint exactly the cis elements involved in amino acid regulation as well as to assess the importance of other promoter elements in transcriptional regulation, we scanned the -150 to +1 region of the AS gene by site-directed mutagenesis. As shown in Fig. 4, each mutation consists of an 8-bp substitution with 2 or $3 \mathrm{bp}$ spacing each of them. Each mutation was introduced in plasmid p3.4CAT and tested for CAT expression under starved and nonstaived conditions (Table 2). Mutations MS1, MS2, MS4, and MS6 had no significant effect on AS gene expression; mutants MS3 and MS5, whose mutations were centered on the Sp1 binding sites, had lower levels of CAT activity but were still regulated to approximately the same extent as the wild type by amino acid starvation. On the other hand, the CAT levels from mutant MS8, whose mutation spanned nucleotides -73 to -66 , were uninducible by amino acid starvation, and its baseline activity was lower than that of the wild type. Mutants MS7, located to the 5' side of MS8, and MS7a, which partially overlaps the MS8 mutation (Fig. 4), were inducible by amino acid starvation. Similar results were observed with mutants MS10 to MS13, although their basal activity was somewhat low. Mutation MS9, located to the $3^{\prime}$ side of MS8, seemed to reduce inducibility. The mutant MS14, mutated around the major transcription start site, had a reduction of CAT gene expression $(6,35)$ but was inducible, confirming that the major transcription start site was not involved in amino acid regulation of the AS gene. These data clearly identify the nucleotides between positions -70 and -66 of the AS promoter as essential for amino acid regulation. Furthermore, it was apparent that none of the 15 mutations tested resulted in a significantly higher and unregulated constitutive

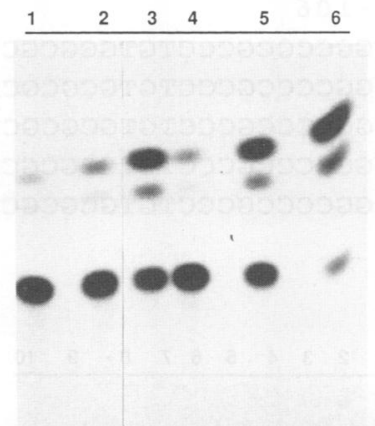

FIG. 5. Evidence that the -73 to -66 region of the AS promoter is essential for amino acid inducibility. (A) Plasmids $\Delta 16$ (lanes 1 and 2), p3.4CAT (lanes 3 and 4), and pSV2CAT (lanes 5 and 6) were transfected into HeLa cells, and CAT activities in cells grown in DMEM containing $10 \%$ dialyzed calf serum (lanes $2,4,6$ ) or DMEM lacking leucine and containing $10 \%$ dialyzed calf serum (lanes 1,3 , 5) were compared. Shown is the autoradiograph of a representative CAT assay thin-layer chromatograph. (B) Plasmids p.0.3CAT (lanes 1 and 2) and p0.3CAT $\Delta 16$ (lanes 3 and 4 ) with the same internal 16 -bp deletion as in plasmid $\Delta 16$ were transfected into HeLa cells, and CAT activities were analyzed as for panel A. Lanes 1 and 3, starved conditions; lanes 2 and 4, nonstarved conditions.

activity, as would be expected from the decreased binding of a factor(s) repressing transcription (23). Rather, the results suggested that the up regulation of AS gene expression by amino acid starvation results from the binding of a positive regulatory element.

To confirm this conclusion, we introduced in the p3.4CAT and p0.3CAT constructs 8- and 16-bp deletions encompassing the region which was mutated in mutant MS8. As shown in Table 2 and Fig. 5, both the 8- and 16-bp deletions in p3.4CAT and the 16-bp deletion in p0.3CAT lowered the basal levels of CAT activity and, as with mutation MS8, completely abolished inducibility; moreover, the mutated AS constructs behaved in response to amino acid starvation as did the control construct pSV2CAT, with their CAT activity being higher under nonstarved conditions.

In a parallel experiment, the p3.4CAT plasmids containing mutations MS1 to MS14 were stably transfected into ts11 cells together with a plasmid conferring G418 resistance. Pools of G418-resistant colonies from each transfection were exposed to $39.5^{\circ} \mathrm{C}$ in the presence or absence of Asn; $16 \mathrm{~h}$ later, CAT activity was determined. Cells transfected with mutant MS8 did not show any regulation of CAT activity in response to Asn deprivation. All other mutants appear to exhibit normal regulation, with the exception of MS9, which also showed reduced inducibility in these experiments (data not shown). Thus, these data indicated that the region of the AS promoter between nucleotides -70 and -64 is an essential cis element for amino acid regulation and tentatively identified this region as the AARE (see below).

Identification of a protein(s) responsible for the induction of AS gene expression by amino acid starvation. Transcriptional regulation is usually achieved through the binding of transcription factors to their target sequences $(25,31)$. We wanted to investigate whether the DNA region centered around nucleotides -71 to -64 was mediating the regulation of AS gene expression through the binding of a protein responsive to amino acid starvation. We therefore designed several overlapping double-stranded oligonucleotides cover- 

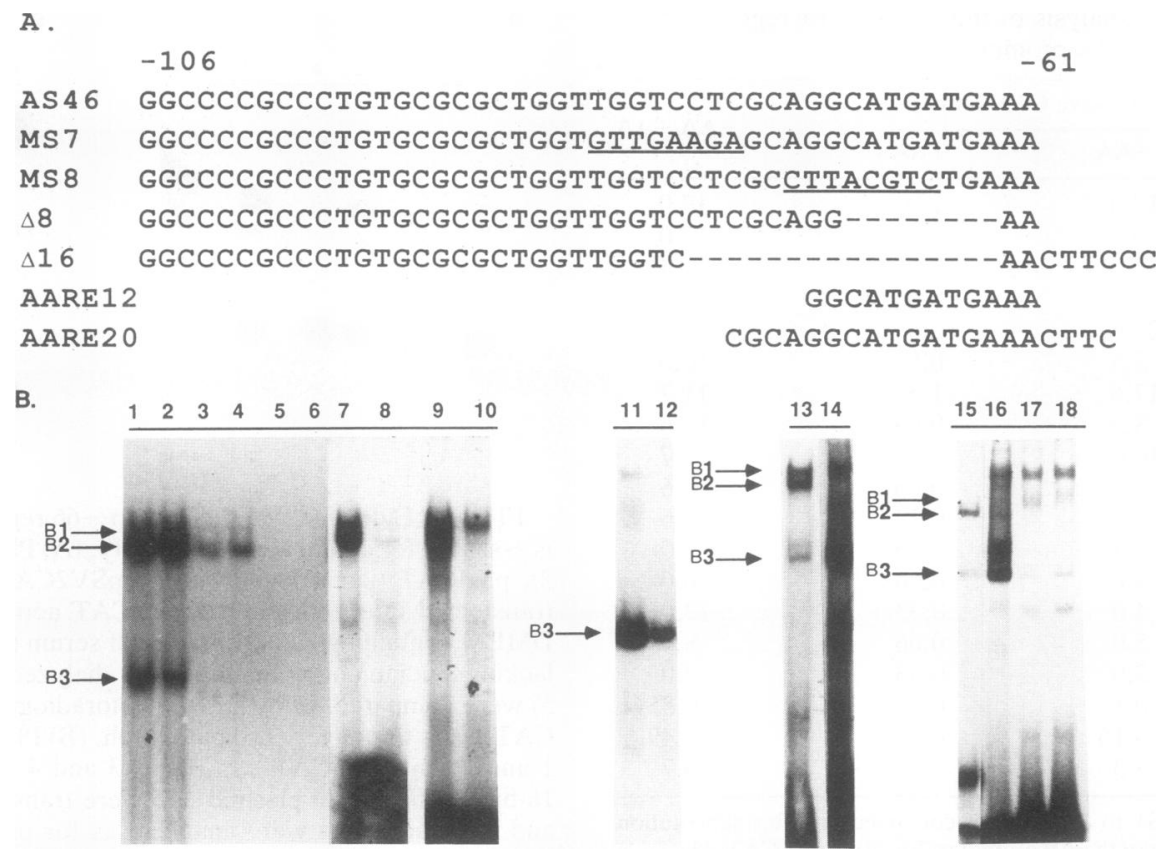

FIG. 6. Proteins binding to the -106 to -55 region of the AS promoter. (A) Sequences of the AS oligonucleotides used in electrophoretic mobility shift assays. Oligonucleotides MS7 and MS8 are identical to AS46 except for a 8-bp substitution (underlined); oligonucleotides $\Delta 8$ and $\Delta 16$ contained internal deletions (dashed line). $\Delta 16$ contained an additional 6 bp $3^{\prime}$ of AS46. AARE12 and AARE20 contained at both $5^{\prime}$ and 3' ends EcoRI and Bam HI recognition sequences, respectively, which are not shown. (B) Electrophoretic mobility shift assays performed with labeled double-stranded AS46 (lanes 1 to 4, 13, and 15), $\Delta 16$ (lanes 5 and 6) $\Delta 8$ (lanes 7 and 8), MS8 (lanes 9 and 10), AARE12 (lanes 11 and 12), MS7 (lane 14), and AARE20 (lanes 16 to 18) oligonucleotides. Nuclear extracts were prepared from HeLa cells grown in nonstarved conditions (lanes 1,3 , and 5 to 18 ) or starved for leucine for $5 \mathrm{~h}$ (lanes 2 and 4). Binding reactions in lanes 3 and 4 were the same as those in lanes 1 and 2, respectively, but were competed for with 100-fold-excess unlabeled AS46. Binding reactions in lanes $6,8,10$, and 12 contained unlabeled competitor $\Delta 16, \Delta 8$, AS46, and AARE12 oligonucleotides, respectively. Binding to AARE 20 (lane 16) was competed for with excess unlabeled AS46 (lane 17) or AARE20 (lane 18). The arrows indicate the major gel retardation bands produced by the AS46 probe, designated B1 to B3.

ing this region and used them for gel retardation assays (Fig. 6). Nuclear extracts from HeLa cells were prepared from nonstarved and amino acid-starved cells for 5 to $6 \mathrm{~h}$, a period of time that is sufficient to induce overexpression of the AS mRNA (data not shown). An oligonucleotide spanning from -65 to -24 did not give any shifted product (not shown), while an oligonucleotide spanning -106 to -61 (AS46; Fig. 6) gave rise to three major retarded bands. Most prominent are two bands migrating close to each other (B1 and B2; Fig. 6B) and a faster-mobility band (B3; Fig. 6B). All of these bands were competed for by excess specific oligonucleotides but not by unrelated DNA sequences. To determine which of the proteins binding to the - 106 to -61 AS DNA region was important for amino acid regulation, we used other oligonucleotides corresponding to the mutations which had been shown to abolish amino acid response in the AS promoter. An oligonucleotide containing the MS8 mutation, that had been shown to abolish amino acid regulation, bound only the proteins corresponding to bands $\mathrm{B} 1$ and $\mathrm{B} 2$, not the protein(s) producing band B3 (Fig. 6B, lane 7). Similar results were obtained with an oligonucleotide containing the internal 8-bp deletion (lane 9). The 16-bp deletion caused loss of binding to the proteins responsible for both slow- and fast-migrating gel shift bands. An oligonucleotide corresponding to mutant MS7 (normal regulation) failed to produce band $\mathrm{B} 2$ but gave rise to normal amounts of the B3 product (Fig. 6B, lane 14). These data seemed to indicate that the protein(s) producing the gel retardation band desig- nated B3 was essential for amino acid regulation and that this protein bound the AS DNA in a region defined by mutation MS8 and by the 8-bp deletion.

To confirm this conclusion, we constructed 12- and 20-bp oligonucleotides, centered on the above-mentioned region (AARE12 and AARE20). Both of these probes bound the protein corresponding to band B3 with high affinity (Fig. 6B, lanes 11 and 16). Furthermore, they competed for the formation of band B3 by the AS46 probe (Fig. 7A). Taken together, these data suggest that the protein giving rise to band $\mathrm{B} 3$ is responsible for amino acid regulation. They also support the hypothesis that up regulation of AS gene expression following amino acid starvation results from binding of a positive regulatory factor, since all mutations which result in low basal activity and lack of amino acid responsiveness abolish binding of the above-mentioned protein. A possible exception is mutant MS9, which appeared to have a reduced level of regulation. An oligonucleotide comprising the MS9 mutation was still capable of binding protein B3 (data not shown). It is possible that this mutation affects binding of an accessory factor or reduces the affinity of binding of protein B3. This possibility is being investigated.

We then wanted to determine whether the abundance of this transcription factor varied with amino acid starvation. For this purpose, nuclear extracts from starved or nonstarved HeLa cells were incubated with the oligonucleotides described above and gel retardation assays were performed. The amount of probe retarded in each of the main bands did 


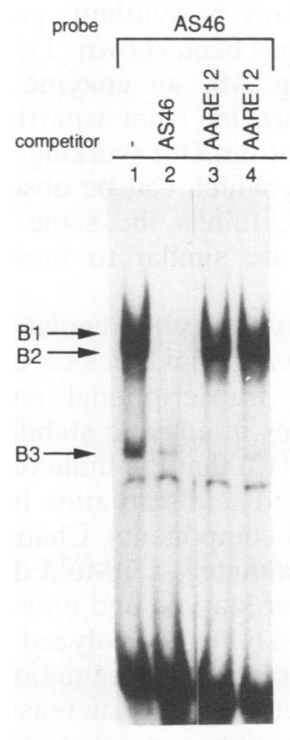

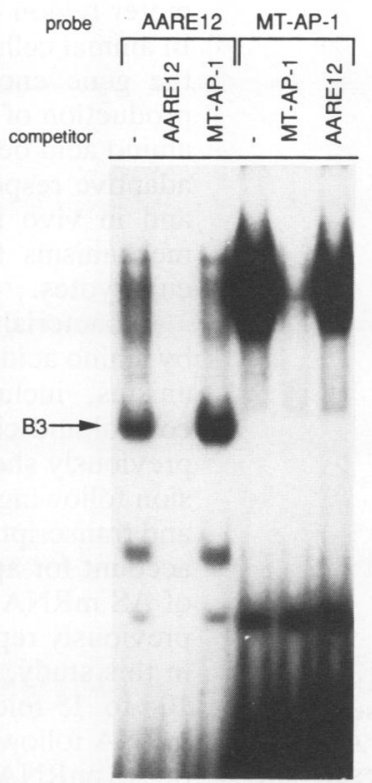

C.

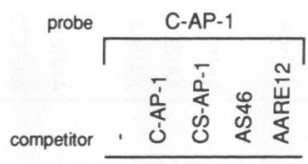

FIG. 7. Comparison of AARE and AP1 binding. (A) Mobility shift analysis using AS46 and AARE12. HeLa nuclear extracts were incubated with ${ }^{32} \mathrm{P}$-labeled AS46 probe in the absence of probe (lane 1) or in the presence of a 200 -fold molar excess of unlabeled AS46 (lane 2) or a 400 -fold (lane 3) or 200-fold (lane 4) excess of AARE12. (B) Competition analysis using AARE12 and AP1 oligonucleotides. Nuclear extracts from nonstarved HeLa cells were incubated with ${ }^{32}$ P-labeled AARE12 or human metallothionein (MT)-AP1 double-stranded oligonucleotide probe in either the absence or presence of 200 -fold-excess unlabeled competitors. (C) Competition analysis using the human collagenase (C)-AP1 oligonucleotide probe in the absence or presence of a 200 -fold molar excess of unlabeled competitors. The oligonucleotide sequences for MT-AP1, C-AP1, and CS-AP1 were 5'-AGCCGCAAGTGACTCAGCGCGGGGCGTG-3'; 5'-TTATAAAG CATGAGTCAGACACCTCT-3', and 5'-AATTCTAGACTGAGTCATGGTACCGAATT-3'; respectively. CS-AP-1 contains the consensus AP1 sequence flanked by random sequences, whereas the MT-AP1 and C-AP1 oligonucleotides represent the naturally occurring sequence. The underlined regions indicate the AP1 binding site. The arrows indicate bands B1 to B3 produced by the AS46 probe.

not seem to vary significantly with amino acid starvation (compare lanes 1 and 2 and lanes 3 and 4 in Fig. 6B). Thus, activation of this factor(s) does not appear to result from changes in its concentration or DNA binding properties (see Discussion), suggesting that secondary modifications activate this protein upon amino acid starvation $(25,31)$. Alternatively, amino acid starvation could induce or alter a noncovalent binding with a second protein that was not detected under our experimental conditions.

The AARE contains some homology to an AP1 binding site but does not bind a classical AP1 complex. Sequence comparison of the region in the AS promoter implicated in amino acid regulation with data base sequences did not give any perfect homology with known transcription factor binding sites; however, the sequence TGATGA contained within the AARE12 sequence showed some homology with the AP1 and GCN4 consensus binding sites (TGA\%TCA and TGACGA). The AP1 transcription complex is composed of two proteins, Fos and Jun, each of which constitutes a family of proteins $(4,7,8,11,26,32)$. Jun is the mammalian homolog of the yeast transcription factor GCN4, the transcriptional activator of many amino acid biosynthetic pathways of the yeast $S$. cerevisiae $(17,18)$. This finding prompted us to investigate whether the protein binding to the AARE could have been AP1 or a related protein. Purified c-Fos and c-Jun proteins ( 1 ; kindly provided by $\mathrm{T}$. Curran) did not bind appreciably our AS46 or AARE12 probes, while the Fos-Jun complex bound with high efficiency to an oligonucleotide (36) containing the consensus AP1 binding site 5'-TGAGTCA-3' (data not shown). This result indicated that a classical Fos-Jun AP1 complex was unlikely to be involved.

We next synthesized two oligonucleotides corresponding to the AP1 binding region of the human metallothionein and collagenase genes, respectively, including $\sim 10$ nucleotides of naturally occurring sequences flanking the AP1 consensus. Figure 7 shows that the major band produced by these probes in gel shift experiments was a broad band, similar in mobility to bands $\mathrm{B} 1$ and $\mathrm{B} 2$ produced by the AS probes. No competition was observed, however, between the AS46 probes and the AP1 probes, indicating that the factors producing bands $\mathrm{B} 1$ and $\mathrm{B} 2$ with the $\mathrm{AS}$ probes are unrelated to an AP1 complex (Fig. 7C and data not shown). No band corresponding in mobility to the $\mathrm{B} 3$ band produced by the AS46 and AARE12 probes was detected with use of the AP1 oligonucleotides (Fig. 7B and C). Accordingly, the AS46 and AARE12 oligonucleotides did not compete appreciably for AP1 binding, although a weak competition was detected with large excesses of cold AARE12 oligonucleotides (Fig. 7C), probably because of the sequence homology mentioned earlier. Thus, these data indicated that the proteins binding to our AS probes in nuclear cell extracts do not correspond to a classical Fos-Jun complex.

To further investigate the significance of the apparent homology between the AARE and AP1 binding sites, we decided to identify the minimum core sequence within the AARE that was capable of binding the protein generating band B3, which previous experiments had identified as the most important for amino acid regulation. For this purpose, we conducted gel shift experiments using a series of mutated 

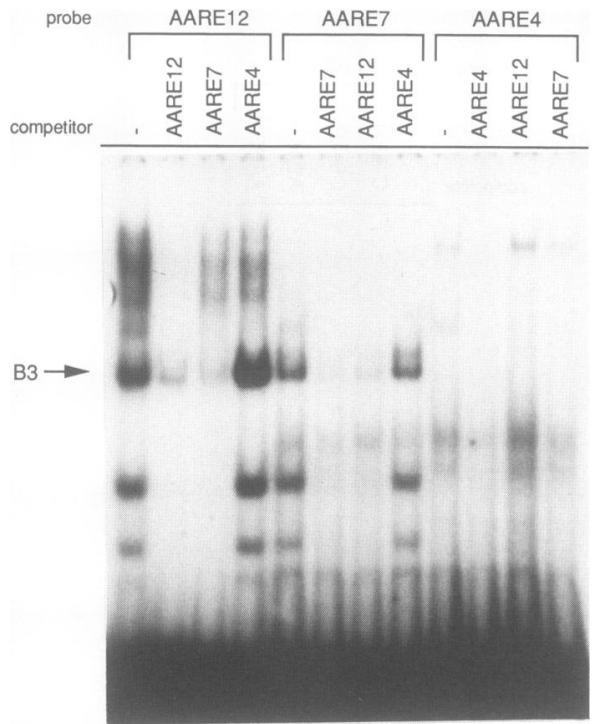

FIG. 8. Identification of the minimum core AARE sequence. Shown is a representative mobility shift analysis using the AARE12 oligonucleotide and two mutated forms. The sequences of the AARE oligonucleotides were 5'-TTGAATTCGGCATGATGAA AGAATCCAA-3' (AARE12), 5'-TTGAATTCTTCATGATGTTCG AATCCAA-3' (AARE7), and 5'-TTGAATTCTTACTGATTCCC GAATCCAA-3' (AARE4). The underlined region indicates the core AS sequence which is flanked by random sequence. Nuclear extracts from nonstarved HeLa cells were incubated with the doublestranded ${ }^{32} \mathrm{P}$-labeled oligonucleotide probes in either the absence or presence of 200 -fold-excess unlabeled competitor. DNA-protein complexes were separated on a native $6 \%$ polyacrylamide gel. The faster-mobility bands are probably a result of protein degradation, as they tend to vary from one nuclear extract preparation to the next. The arrow indicates the major band (B3) produced by the AARE probes.

AARE12 oligonucleotides. Figure 8 shows that the AARE7 probe, which contains the sequence CATGATG, is still capable of binding the protein producing B3. Further base substitutions from either end of this sequence abolished or greatly reduced binding (Fig. 8 and data not shown). These results therefore established that the minimum core sequence within the AARE capable of binding the protein producing B3 is $5^{\prime}$-CATGATG-3'. This sequence is significantly different from the AP1 consensus (TGAG/cTCA), although it is also a palindromic sequence.

From these data, we conclude that the proteins involved in the regulation of the AS promoter are unlikely to be part of the AP1 complex, although it is possible that they may be related.

\section{DISCUSSION}

Changes in gene expression in response to variations in intracellular amino acids levels take place in prokaryotes and in lower eukaryotes $(17,29)$. The Escherichia coli tryptophan operon has been shown to modulate its expression in response to the levels of charged tRNA ${ }^{\text {Trp }}$. In the yeast $S$. cerevisiae, amino acid starvation induces the synthesis of the enzymes required for amino acid biosynthesis through posttranscriptional activation of the transcription factor GCN4. Starvation conditions activate GCN4 by preferential usage of the correct open reading frame for its translation product. Transactivation then occurs through the binding of
GCN4 to its target sequences that are present in the promoter region of many biosynthetic pathway genes $(17,18)$. In animal cells, it has been shown $(13,15)$ that expression of the gene encoding AS, an enzyme which catalyzes the production of asparagine from aspartic acid, is activated by amino acid deprivation. Our working hypothesis is that this adaptive response, which can be observed in vitro $(13,15)$ and in vivo (15a), fulfills the same function and utilizes mechanisms that are similar to those operating in lower eukaryotes.

In bacterial and yeast cells, regulation of gene expression by amino acid starvation involves several coordinated mechanisms, including transcriptional and posttranscriptional control and changes in enzyme stability. Similarly, we had previously shown (13) that the induction of AS gene expression following amino acid starvation had posttranscriptional and transcriptional components. Changes in mRNA stability account for approximately a 10-fold difference in the levels of AS mRNA under starved and nonstarved conditions. As previously reported (13) and analyzed in much greater detail in this study, transcriptional regulation contributes another 10 - to 15-fold factor to the increased expression of AS mRNA following amino acid deprivation, resulting in levels of AS mRNA which are almost 100 -fold higher in starved versus nonstarved cells $(13,15)$. We had also shown (13) that expression of AS mRNA was regulated not only by Asn but also by variations in the concentration of several other essential amino acids, suggesting common mechanism(s) of regulation that are likely to operate on other genes of the biosynthetic pathways.

The work presented in this report focuses on the identification of the cis- and trans-acting elements which are involved in the transcriptional regulation of AS gene expression. By constructing plasmids in which CAT gene expression was under the control of the AS $5^{\prime}$ region and transfecting them into cells under starved or normal conditions, we mapped the AS promoter to a region between nucleotide -164 and the major transcription start site (designated +1 ). This DNA fragment contains two putative Sp1 binding sites, which are important for the basal level of expression of its gene but are not involved in amino acid regulation.

Other components of this promoter are a TATAA sequence which appears to direct transcription initiation about $30 \mathrm{bp}$ downstream and a number of minor initiation sites upstream of the major one. The relative utilization of these sites does not change with amino acid concentration (data not shown), and we could show that the TATAA sequence and the sequences around the major initiation site are not involved in amino acid regulation. A surprising finding was the presence, downstream of the major initiation site and within the untranslated first exon, of sequences that appear to decrease the basal activity of the AS 5 ' region. This region has significant homology to the FIRE sequence (22), a negative regulatory element contained in comparable location in the first exon of the fos gene. This AS region appears to bind at least two single-stranded-DNA-binding proteins. Mutations that abolish binding of these proteins considerably elevate basal activity of the AS CAT plasmids but do not affect amino acid regulation. The significance of this finding remains to be investigated. The physiological functions of these proteins, which can bind only to the coding strand of the AS DNA, could actually be unrelated to transcription, or they could exert some steric hindrance on transcription elongation.

The cis DNA elements which are important for amino acid regulation of the AS promoter map to a stretch of nucle- 
otides located approximately $60 \mathrm{bp}$ upstream of the major transcription initiation site. Mutations or deletions affecting this sequence, which we have designated the AARE, result in low and unregulated activity of the AS promoter, indicating that up regulation of AS gene expression following amino acid starvation results from binding of a positive factor rather than removal of a repressor-like protein. We have not yet been able to transfer amino acid responsiveness to a heterologous promoter by inserting the AARE12, AARE20, or AS46 sequences next to it. We have inserted the same oligonucleotides at the SacI site of the $\Delta 8$-CAT plasmid (which has low and unregulated activity) and observed some increase in activity and a slight evidence of amino acid regulation. It is likely that the AARE has positional requirements, and we are therefore continuing these experiments in an attempt to define these requirements.

The sequences around and comprising the AARE bind at least three proteins in gel shift assays. Of these, the most significant appears to be the protein generating the fastestmobility band (B3), as its binding to the AS promoter correlates with the presence of an intact AARE. However, no differences in the abundance or in the DNA binding properties of this protein were observed in HeLa nuclear cell extracts from starved or nonstarved cells; posttranscriptional modifications of this protein which do not affect binding to its DNA target site may be induced by amino acid starvation. Transcription factors are believed to be modular proteins composed of an activation domain and a DNA binding domain $(12,25)$. While the DNA binding domains have a very high specificity for their target sequences, the transactivating domains are less specific, being generally characterized by a rather high content of acidic residues (12, $25,31)$. Transcription factors binding to the target site can be regulated by posttranslational modification. Phosphorylation of the DNA binding domain of the Jun protein has been shown to inhibit Jun binding to its target sequence (5), and other phosphorylation events may also modulate Jun action (2). Posttranslational modifications can also influence the activity of the protein transactivating domain without affecting its DNA binding capabilities (11). Glycosylation of the transcription factor $\mathrm{Sp} 1$ has been demonstrated to be required for transactivation to occur, while in its absence, the DNA binding properties of the protein do not change (19). It is also possible that the protein binding to the AARE is complexed with another protein, whose activity or synthesis is induced by amino acid starvation, but the complex is labile and does not survive the rather crude manipulation of a gel shift assay.

The AARE region contains a stretch of nucleotides showing some homology to AP1 or GCN4 binding sites. This observation prompted us to examine whether the protein(s) binding to the AARE could have been related to AP1. It is known that a wide range of dimeric complexes can be generated among members of the Fos-Jun and ATF-CREB protein families and that they display different DNA binding specificities $(8,11,20,27)$. The results obtained, however, indicate that the AARE does not bind an AP1 complex. Although a weak competition for AP1 binding can be observed with a large excess of AARE oligonucleotides, this competition is not likely to have physiological implications and probably reflects only sequence similarity. This conclusion is supported by the finding that gel shift experiments with the AP1 probes did not produce any band corresponding to the B3 band obtained with our AS probes, whose presence had been identified as strictly correlated with amino acid regulation of the AS promoter. Furthermore, the minimum core sequence capable of binding the protein generating band B3 is CATGATG, quite distinct from the AP1 consensus.

It is interesting to note that the minimum AARE core sequence binding the protein producing B3 is located immediately $5^{\prime}$ of the sequence mutated in mutant MS9, which had a reduced level of regulation. Although an oligonucleotide containing the MS9 mutation appears to bind the protein producing B3 normally, it is possible that in vivo the stability or the affinity of this interaction is reduced.

In conclusion, we have shown in this report that the transcriptional regulation of AS gene expression by amino acid concentration involves the binding of a positive regulatory factor to a sequence located about 60 nucleotides upstream of the major transcription initiation site. The mechanism of activation of this factor(s) seems to involve posttranslational modifications or complexing with other proteins, as its abundance does not seem to be influenced by amino acid starvation. Further work will be required to identify precisely the trans-acting factor(s) involved in the regulation of the AS promoter as well as the mechanism of its activation by amino acid starvation.

\section{ACKNOWLEDGMENTS}

We thank Ottavia Barbieri for helping in some of these experiments and David Levy for suggestion and advice. We also thank Tom Curran for providing purified Fos and Jun proteins and antibodies and R. Bravo and D. Nathans for anti-Jun and Fos antisera.

This investigation was supported by PHS grant CA42568 from the National Cancer Institute.

\section{REFERENCES}

1. Abate, C., D. Luk, and T. Curran. 1990. An ubiquitous nuclear protein stimulates the DNA-binding activity of fos and jun indirectly. Cell Growth Differ. 1:455-462.

2. Adler, V., C. C. Franklin, and A. S. Kraft. 1992. Phorbol esters stimulate the phosphorylation of c-jun but not v-jun: regulation by the N-terminal $\delta$ domain. Proc. Natl. Acad. Sci. USA 89:5341-5345.

3. Anderson, G. M., and S. O. Freytag. 1991. Synergistic activation of a human promoter in vivo by transcription factor Sp1. Mol. Cell. Biol. 11:1935-1943.

4. Bohmann, D., T. J. Bos, A. Admon, T. Nishimura, P. K. Vogt, and R. Tjian. 1987. Human proto-oncogene c-jun encodes a DNA binding protein with structural and functional properties of transcription factor AP-1. Science 238:1386-1392.

5. Boyle, W. J., T. Smeal, L. H. K. Defize, P. Angel, J. R. Woodgett, M. Karin, and T. Hunter. 1991. Activation of protein kinase $\mathrm{C}$ decreases phosphorylation of $\mathrm{c}$ jun at sites that negatively regulated its DNA-binding activity. Cell 64:573-584.

6. Carcamo, J., L. Buckbinder, and D. Reinberg. 1991. The initiator directs the assembly of a transcription factor IID-dependent transcription complex. Proc. Natl. Acad. Sci. USA 88:80528056.

7. Chiu, R., W. J. Boyle, J. Meek, T. Smeal, T. Hunter, and M. Karin. 1988. The c-fos protein interacts with c-jun/AP-1 to stimulate transcription of AP-1 responsive genes. Cell 54:541552.

8. Curran, T., and B. R. Franza, Jr. 1988. Fos and jun: the AP-1 connection. Cell 55:395-397.

9. Darnell, J., H. Lodish, and D. Baltimore. 1986. Gene control in eukaryotes, p. 465-575. Molecular cell biology. W. H. Freeman \& Co., New York.

10. Dignam, J. D., R. M. Lebovitz, and R. G. Roeder. 1983. Accurate transcription initiation by RNA polymerase II in a soluble extract from isolated mammalian nuclei. Nucleic Acids Res. 11:1475-1489.

11. Forrest, D., and T. Curran. 1992. Crossed signals: oncogenic transcription factors. Curr. Opin. Genet. Dev. 2:19-27. 
12. Frankel, A. D., and P. S. Kim. 1991. Modular structure of transcription factors: implications for gene regulation. Cell 65:717-719.

13. Gong, S. S., L. Guerrini, and C. Basilico. 1991. Regulation of asparagine synthetase gene expression by amino acid starvation. Mol. Cell. Biol. 11:6059-6066.

14. Gorman, C. M., L. F. Moffat, and B. H. Howard. 1982. Recombinant genomes which express chloramphenicol acetyltransferase in mammalian cells. Mol. Cell. Biol. 2:1044-1051.

15. Greco, A., S. S. Gong, M. Ittman, and C. Basilico. 1989. Organization and expression of the cell cycle gene, $t s 11$, that encodes asparagine synthetase. Mol. Cell. Biol. 9:2350-2359.

15a.Guerrini, L., O. Barbieri, and C. Basilico. Unpublished data.

16. Hall, C. V., P. E. Jacob, G. M. Ringold, and F. Lee. 1983. Expression and regulation of Escherichia coli lacZ gene fusions in inammalian cells. J. Mol. Appl. Genet. 2:101-109.

17. Hope, I. A., and K. Struhl. 1985. GCN4 protein, synthesized in vitro, binds HIS 3 regulatory sequences: implications for general control of amino acid biosynthetic genes in yeast. Cell 43:177188.

18. Hope, I. A., and K. Struhl. 1987. GCN4, a eukaryotic transcriptional activator protein, binds as a dimer to target DNA. EMBO J. 6:2781-2784.

19. Jackson, S. P., and R. Tjian. 1988. O-glycosylation of eukaryotic transcription factors: implications for mechanisms of transcriptional regulation. Cell 55:125-133.

20. Kouzarides, T., and E. Ziff. 1989. Leucine zippers of fos, jun and GCN4 dictate dimerization specificity and thereby control DNA binding. Nature (London) 340:568-571.

21. Kunkel, T. A. 1985. Rapid and efficient site-specific mutagenesis without phenotypic selection. Proc. Natl. Acad. Sci. USA 82:488-492.

22. Lamb, N. J. C., A. Fernandez, N. Tourkine, P. Jeanteur, and J.-M. Blanchard. 1990. Demonstration in living cells of an intragenic negative regulatory element within the rodent $c$-fos gene. Cell 61:485-496.

23. Levine, M., and J. L. Manley. 1989. Transcriptional repression of eukaryotic promoters. Cell 59:405-408.

24. Melton, D. A., P. A. Krieg, M. R. Rebagliati, T. Maniatis, K. Zinn, and M. R. Green. 1984. Efficient in vitro synthesis of biologically active RNA and RNA hybridization probes from plasmids containing a bacteriophage SP6 promoter. Nucleic Acids Res. 12:7035-7056.

25. Mitchell, P. J., and R. Tjian. 1989. Transcriptional regulation in mammalian cells by sequence-specific DNA binding proteins. Science 245:371-378.

26. Müller, R. 1989. The leucine repeat motif in fos protein mediates complex formation with jun/AP-1 and is required for transformation. Cell 56:507-516.

27. Nakabeppu, Y., K. Ryder, and D. Nathans. 1988. DNA binding activities of three murine jun proteins: stimulation by fos. Cell 55:907-915.

28. Peritz, L. N., E. J. B. Fodor, D. W. Silversides, P. A. Cattini, J. D. Baxter, and N. L. Eberhardt. 1988. The human growth hormone gene contains both positive and negative control elements. J. Biol. Chem. 263:5005-5007.

29. Platt, T. 1990. Regulation of gene expression in the tryptophan operon of Escherichia coli. Mol. Cell. Biol. 10:263-299.

30. Pohjanpelto, P., and E. Hölttä. 1990. Deprivation of a single amino acid induces protein synthesis-dependent increases in c-jun, c-myc, and ornithine decarboxylase mRNAs in Chinese hamster ovary cells. Mol. Cell. Biol. 10:5814-5821.

31. Ptashne, M. 1988. How eukaryotic transcriptional activators work. Nature (London) 335:683-689.

32. Rauscher, F. J., III, L. C. Sambucetti, T. Curran, R. J. Distel, and B. M. Spiegelman. 1988. Common DNA binding site for fos protein complexes and transcription factor AP-1. Cell 52:471480.

33. Sambrook, J., E. F. Fritsch, and T. Maniatis. 1989. Molecular cloning: a laboratory manual. Cold Spring Harbor Laboratory, Cold Spring Harbor, N.Y.

34. Sanger, R., S. Nicklen, and A. R. Coulson. 1977. DNA sequencing with chain-terminating inhibitors. Proc. Natl. Acad. Sci. USA 74:5463-5467.

35. Smale, S. T., and D. Baltimore. 1989. The "initiator" as a transcription control element. Cell 57:103-113.

36. Velcich, A., and E. B. Ziff. 1990. Functional analysis of an isolated fos promoter element with AP-1 site homology reveals cell type-specific transcriptional properties. Mol. Cell. Biol. 10:6273-6282. 